\title{
Corticotropin-Releasing Hormone Receptor Type 1 (CRHR1) Genetic Variation and Stress Interact to Influence Reward Learning
}

\author{
Ryan Bogdan, ${ }^{1}$ Diane L. Santesso, ${ }^{1}$ Jesen Fagerness, ${ }^{2}$ Roy H. Perlis, ${ }^{2,3}$ and Diego A. Pizzagalli1 ${ }^{1,4}$ \\ ${ }^{1}$ Department of Psychology, Harvard University, Cambridge, Massachusetts 02138, ${ }^{2}$ Center for Human Genetic Research, Massachusetts General Hospital, \\ and ${ }^{3}$ Bipolar Clinical and Research Program, Massachusetts General Hospital and Harvard Medical School, Boston, Massachusetts 02114, and ${ }^{4}$ Center For \\ Depression, Anxiety and Stress Research and Neuroimaging Center, McLean Hospital and Harvard Medical School, Belmont, Massachusetts 02478
}

Stress is a general risk factor for psychopathology, but the mechanisms underlying this relationship remain largely unknown. Animal studies and limited human research suggest that stress can induce anhedonic behavior. Moreover, emerging data indicate that genetic variation within the corticotropin-releasing hormone type 1 receptor gene (CRHR1) at rs12938031 may promote psychopathology, particularly in the context of stress. Using an intermediate phenotypic neurogenetics approach, we assessed how stress and CRHR1 genetic variation ( $\mathrm{rs} 12938031)$ influence reward learning, an important component of anhedonia. Psychiatrically healthy female participants $(n=75)$ completed a probabilistic reward learning task during stress and no-stress conditions while 128-channel event-related potentials were recorded. Fifty-six participants were also genotyped across CRHR1. Response bias, an individual's ability to modulate behavior as a function of reward, was the primary behavioral variable of interest. The feedback-related positivity (FRP) in response to reward feedback was used as a neural index of reward learning. Relative to the no-stress condition, acute stress was associated with blunted response bias as well as a smaller and delayed FRP (indicative of disrupted reward learning) and reduced anterior cingulate and orbitofrontal cortex activation to reward. Critically, rs12938031 interacted with stress to influence reward learning: both behaviorally and neurally, A homozygotes showed stress-induced reward learning abnormalities. These findings indicate that acute, uncontrollable stressors reduce participants' ability to modulate behavior as a function of reward, and that such effects are modulated by CRHR1 genotype. Homozygosity for the A allele at rs12938031 may increase risk for psychopathology via stress-induced reward learning deficits.

\section{Introduction}

Progress toward a better understanding of psychopathology is hindered by substantial clinical heterogeneity. Focusing on intermediate phenotypes hypothesized to lie between genetic/envi-

Received May 27, 2011; revised July 11, 2011; accepted July 21, 2011.

Author contributions: R.B. and D.A.P. designed research; R.B. performed research; R.B., D.L.S., J.F., R.H.P., and D.A.P. contributed unpublished reagents/analytic tools; R.B., D.L.S., J.F., R.H.P., and D.A.P. analyzed data; R.B., D.L.S., J.F., R.H.P., and D.A.P. wrote the paper.

This work was supported by Sackler Scholar in Psychobiology (Harvard University) and Mind, Brain, and Behavior (Harvard University) grants awarded to R.B., and by NIMH Grants R01 MH68376 and R21 MH078979, Merck Awards for Genome-Related Research (Harvard University), Talley Fund (Harvard University) grants, and a 2008 National Alliance for Research on Schizophrenia and Depression Independent Investigator Award (D.A.P.). We thank Brian Galloway, Miles Nugent, Avram Holmes, Yuliya Nikolova, Sara Rubenstein, Courtney Bagge, Scott Coffey, and Yan Emily Yuan for their assistance. Parts of this paper have been presented previously at the 22nd Annual Meeting of the Society for Research in Psychopathology (September 2008; Pittsburgh, PA), the 23rd Annual Meeting of the Society for Research in Psychopathology (September 2009; Minneapolis, MN), and the 49th Annual Meeting of the Society for Psychophysiological Research (October 2009; Berlin, Germany).

D.A.P. has received consulting fees from ANT North America Inc. (Advanced Neuro Technology) and AstraZeneca, and honoraria from AstraZeneca. R.H.P. has received honoraria and speaker's or consulting fees from AstraZeneca, Bristol-Myers Squibb, Eli Lilly and Company, GlaxoSmithKline, Pfizer, and Proteus. R.H.P. is a major stockholder in Concordant Rater Systems, LLC. R.B., D.L.S., and J.F. declare no competing financial interests.

Correspondence should be addressed to Diego A. Pizzagalli, Center for Depression, Anxiety and Stress Research, McLean Hospital, 115 Mill Street, Belmont, MA 02478. E-mail: dap@mclean.harvard.edu.

R. Bogdan's present address: Department of Psychology and Neuroscience, Duke University, Box 90086, 417 Chapel Drive, Durham, NC 27708.

DOI:10.1523/JNEUROSCI.2661-11.2011

Copyright $\odot 2011$ the authors $\quad 0270-6474 / 11 / 3113246-09 \$ 15.00 / 0$ ronmental risk factors and disease provides a powerful alternative (Gottesman and Gould, 2003). Anhedonia, the loss of pleasure, is a promising depressive phenotype (Hasler et al., 2004), particularly in the context of Gene by Environment interaction because (1) hedonic behavior is heritable and genetically associated with stress (Bogdan and Pizzagalli, 2009); (2) genetic variants affect reward processing (Dreher et al., 2009); and (3) stress can induce anhedonic behavior and dysfunction within reward-related pathways (Berenbaum and Connelly, 1993; Anisman and Matheson, 2005; Bogdan and Pizzagalli, 2006; Dillon et al., 2009).

As a regulator of the hypothalamic-pituitary-adrenal (HPA) axis, the corticotropin-releasing hormone $(\mathrm{CRH})$ system plays a pivotal role in stress responsiveness. Manipulations that reduce corticotropin-releasing hormone type 1 receptor (CRHR1) function lower HPA axis responses to stress, promote approach, and modulate dopamine function (Takahashi, 2001; Hauger et al., 2006; Beckstead et al., 2009; Binder and Nemeroff, 2010). Critically, the A allele of a single-nucleotide polymorphism (SNP) within CRHR1 (rs12938031; A/G) has been associated with the following: enhanced CRHR1 mRNA expression, diminished HPA response to CRH infusion (mimicking patterns observed in depression), elevated depression after childhood trauma, and blunted ventral striatal reactivity to positive feedback (Thode et al., 2011). Given that (1) depression is characterized by reward processing deficits (Pizzagalli et al., 2005, 2008), (2) stress dis- 
rupts reward processing (Berenbaum and Connelly, 1993; Bogdan and Pizzagalli, 2006), and (3) the A allele is associated with blunted neural responses to positive feedback (Thode et al., 2011), the A allele may increase risk for depression via stressinduced reward processing deficits.

The goal of this study was to assess how stress and CRHR1 genotype (rs12938031) influence an important behavioral component of anhedonia: reward learning. To this end, 75 healthy Caucasian females completed a probabilistic reward learning task during acute stress (threat of shock) and no-stress conditions while event-related potentials (ERPs) were recorded. ERP analyses focused on the feedback-related positivity (FRP), a frontocentral positive-going deflection elicited by positive prediction errors and rewards, hypothesized to originate from dorsal anterior cingulate cortex (ACC) and striatal regions implicated in reinforcement learning (Potts et al., 2006; Eppinger et al., 2008; Holroyd et al., 2008; Foti et al., 2011). Critically, reduced learning in probabilistic learning paradigms has been linked to a smaller FRP (or larger feedback-related negativity) to positive feedback (Müller et al., 2005; Santesso et al., 2008).

Owing prior research (Bogdan and Pizzagalli, 2006), we hypothesized that stress would reduce reward learning. Moreover, based on findings that blunted reward learning is associated with smaller FRP to rewards (Müller et al., 2005; Santesso et al., 2008), and ACC regions are critically implicated in the integration of reinforcement history (Kennerley et al., 2006), we expected that stress would lead to a reduced FRP and ACC activation to reward feedback. Finally, we hypothesized that A homozygotes at rs12938031 would be particularly susceptible to stress-induced reward learning deficits.

\section{Materials and Methods Participants}

Eighty-four healthy female participants aged 18-25 were recruited from the community. All participants were right-handed and free of color blindness, past or present neurological, psychiatric, hormonal, or metabolic disturbances. To limit potential confounds of population stratification, only individuals of European descent (i.e., with two parents of European ancestry) were recruited. Only females were included because women are more likely than men to suffer from depression and gender differences in behavioral and physiological responses to stress are theorized to contribute to this difference (Nolen-Hoeksema et al., 1999). Seventy-five participants (age, mean $\pm \mathrm{SD}, 21.88 \pm 1.97$; education, $15.42 \pm 1.46$ years) were available for behavioral analyses. [Of the original 84 participants, 9 were excluded because of technical difficulties $(n=$ $3)$, task noncompliance $(n=2)$, or below chance task performance $(n=$ 4).] The FRP could be reliably identified in both conditions (see below) in 56 of these participants, who were considered for ERP analyses [FRP data were unavailable in 19 subjects due to technical difficulties $(n=3)$ and poor data quality (i.e., poor signal-noise ratio upon visual inspection resulting in unidentifiable components) in one $(n=15)$ or both $(n=1)$ conditions]. Genetic data for 19 subjects were lost due to human error when genetic storage facilities moved to another building, and genotyping rs12938031 failed for two participants, leaving a total of 54 participants with genetic, behavioral, and ERP data (41 of whom had FRP data). Participants provided written informed consent to a protocol approved by the Committee on the Use of Human Subjects at Harvard University. Participants were paid \$10/h for their time and "won" \$15 during the reward task.

\section{Procedure}

The study consisted of two sessions. In the first, the Structured Clinical Interview for the DSM-IV (SCID) (First et al., 2002) was administered to ensure that participants had no past or present axis I disorders. Participants then completed several self-report measures assessing mood and stress, including the Mood and Anxiety Symptom Questionnaire (MASQ) (Watson et al., 1995) and Perceived Stress Scale (PSS) (Cohen et al., 1983) and provided a saliva sample for DNA analysis (Oragene; DNA Genotek). In the second session, participants performed the probabilistic reward task while 128-channel ERPs were recorded and completed the Beck Depression Inventory II (Beck et al., 1996). Session 2 was completed within $14 \mathrm{~d}$ of Session $1(5.71 \pm 4.16 \mathrm{~d})$.

\section{Probabilistic reward task}

A probabilistic reward task (Pizzagalli et al., 2005) was administered to objectively measure reward learning (i.e., an individual's propensity to modulate behavior according to reinforcement history). The task was adapted from prior research (Tripp and Alsop, 1999) and has been validated in multiple samples. Importantly, reward learning as measured by this task is (1) blunted in depressed subjects (Pizzagalli et al., 2005, 2008), (2) reduced under stress in healthy controls (Bogdan and Pizzagalli, 2006), (3) 48\% heritable and genetically associated with stress perception (Bogdan and Pizzagalli, 2009), and (4) associated with increased dorsal ACC and basal ganglia activation as well as larger FRP to reward feedback (Santesso et al., 2008).

In each condition (stress, no-stress), participants completed three blocks of 80 trials (240 trials total). During each trial, participants identified whether a mouth or nose was long (mouth, $11.00 \mathrm{~mm}$; nose, $5.31 \mathrm{~mm}$ ) or short (mouth, $10.00 \mathrm{~mm}$; nose, $5.00 \mathrm{~mm}$ ) by pressing a corresponding response key. Importantly, the small size difference between stimuli and short stimulus exposure (100 ms) made it difficult to ascertain which stimulus was presented. Critically, an asymmetric reinforcement schedule was used to induce a response bias. Specifically, in each block, correct identification of either the short or long stimulus ("rich stimulus") was rewarded ("Correct!! You won 5 cents") three times more frequently than the other stimulus ("lean stimulus"). Under these contingencies, both human and nonhuman animals develop a response bias toward the more frequently rewarded ("rich") stimulus (McCarthy and Davison, 1979). The stimulus disproportionally rewarded and response key assignments were counterbalanced across participants.

To ensure similar reward ratio exposure across subjects and conditions, only 32 correct trials ( 24 rich, 8 lean) were scheduled to receive reward in each block. Reward was administered according to a controlled reinforcer procedure; if a participant was incorrect on a trial that was scheduled to be reinforced, reward feedback was delayed until the next correct identification of the same stimulus type. In addition, a pseudorandomized reinforcement sequence was used with the constraint that no stimulus could be presented more than three times in a row. Before the experiment, participants were informed that their goal was to win as much money as possible and that not all correct responses would be rewarded. No information was provided regarding the differential reinforcement schedule.

In addition to standard measures of hit rate and reaction time (RT), this task yields measures of "discriminability," which indexes the ability to perceptually distinguish two stimuli, and "response bias," which reflects the tendency to select one stimulus regardless of actual stimulus presentation and was our primary behavioral variable of interest.

\section{Stress manipulation}

Participants completed the task during stress and no-stress conditions (order counterbalanced across subjects) while skin conductance and ERPs were recorded. Threat of shock, which has been shown to induce negative affect, anxiety, and stress responses as well as reduce reward learning (Grillon et al., 1993; Bogdan and Pizzagalli, 2006), was used as the acute stressor. Before the stress condition, a shock device (model E13-22; Coulbourn Instruments) was introduced into the room and electrodes were attached to the back of the participant's right hand, $0.5 \mathrm{~cm}$ apart. Before the stress condition, shock was individually titrated by increasing intensity (starting at $0.4 \mathrm{~mA}$ ) until the participant subjectively defined it as "highly aversive or unpleasant, but not painful" or it reached $4.0 \mathrm{~mA}(2.22 \pm 0.85 \mathrm{~mA})$. 
Participants were instructed that they would receive one to three electrical shocks while completing the stress condition. Additionally, because uncontrollability of stress has been associated with an enhanced stress response and anhedonic behavior across species (Dickerson and Kemeny, 2004; Anisman and Matheson, 2005), participants were told that shocks were randomly generated by the computer and were in no way linked to their performance. Four additional trials (trials 81-84) were added to the end of the first block in each condition (excluded from the analyses). All participants received a $1 \mathrm{~s}$ shock during trial 81 of the stress condition. In an effort to maintain stress throughout the experiment, following the conclusion of the second block, the experimenter (R. Bogdan) informed the participant: "I am aware you did not receive a shock during the last block of the task. As a result, it is highly likely that you will receive a shock during the next block." Before the no-stress condition, participants were informed that it was impossible to receive a shock. To reinforce these instructions, the shock device was never introduced into the room (if the no-stress condition was first) or was removed for at least 10 min before the no-stress condition (if the no-stress condition was second).

Visual analog scales (relaxed-tense; anxious-at ease) were administered before each condition and following the 10 min break. Visual Analog Mood Scale (VAMS) ratings were used to ensure that the second condition began only after participants' mood had returned to baseline levels (if necessary, an additional rest period was provided). To assess the effectiveness of the stress manipulation, skin conductance was collected and state-related mood measures, the State-Trait Anxiety Inventory (STAI) (Spielberger et al., 1970) and Positive and Negative Affect Schedule (PANAS) (Watson et al., 1988), were given before and after each administration of the probabilistic reward task to assess state anxiety and affect, respectively (one participant did not complete these scales and was excluded from analyses with these variables). Posttask scales were modified to ask participants about mood while they were completing the task.

\section{Behavioral, physiological, and genetic data collection and reduction}

\section{Behavioral data}

The behavioral task was presented with E-prime software (version 1.2; Psychology Software Tools). A two-step procedure previously described was used to identify outlier responses (Pizzagalli et al., 2005). First, trials with RTs $<100$ or $>1500$ ms were excluded. Second, for each subject, trials with RTs (following natural log transformation) falling outside the mean \pm 3 SDs were removed. Following outlier removal, response bias (the main variable of interest) and discriminability were computed as follows (Pizzagalli et al., 2007; Bogdan et al., 2010):

Response bias:

$$
\log b=\frac{1}{2} \log \left(\frac{\left(\text { Rich }_{\text {correct }}+0.5\right) *\left(\text { Lean }_{\text {incorrect }}+0.5\right)}{\left(\text { Rich }_{\text {incorrect }}+0.5\right) *\left(\text { Lean }_{\text {correct }}+0.5\right)}\right)
$$

Discriminability:

$$
\log d=\frac{1}{2} \log \left(\frac{\left(\text { Rich }_{\text {correct }}+0.5\right) *\left(\operatorname{Lean}_{\text {correct }}+0.5\right)}{\left(\text { Rich }_{\text {incorrect }}+0.5\right) *\left(\text { Lean }_{\text {incorrect }}+0.5\right)}\right) .
$$

Consistent with prior recommendations (Hautus, 1995), 0.5 was added to every cell within the formula to allow for the computation of response bias and discriminability in cases that have a zero in one cell of the formula.

\section{Skin conductance}

Electrodes (Kendall Foam 4103, Tyco Healthcare Group) were applied to the first and second finger tip of the left hand to measure skin conductance. Psylab hardware [Stand Alone Monitor (SAM); SC5 SC Coupler] and software (Psylab8; Psylab Measurement and Analysis Software) were used for the collection, measurement, and analysis of skin conductance data (Psylab; Contact Precision Instruments). Recording was conducted at $300 \mathrm{~Hz}$ with default filters ( $10 \mathrm{~Hz}$ low pass). Data were resampled at 10 $\mathrm{Hz}$. The onset, slope, and peak were used to identify each nonspecific response to avoid the accidental detection of responses due to noise or movement.

\section{ERP}

EEG was recorded using a 128-channel sensor net (Electrical Geodesics) and sampled at $500 \mathrm{~Hz}$ (16-bit precision; bandwidth, 0.01-100 Hz; impedances, $<45 \mathrm{k} \Omega$ ) referenced to the vertex. Data were then resampled to $250 \mathrm{~Hz}$ and gross artifacts were manually removed. BrainVision software was used for ERP analyses (Brain Products). Spatially weighted linear interpolations were used to replace noisy channels and an independent component analysis was applied to correct for eye and electrocardiogram artifacts (Jung et al., 2000). For each block, EEG epochs were extracted $200 \mathrm{~ms}$ before and $800 \mathrm{~ms}$ after reward feedback for correct identification of the rich and lean stimuli (i.e., pooled across stimuli). An automatic artifact removal ( $\pm 75 \mu \mathrm{V}$ criterion) was then applied to identify any remaining artifacts. Next, data were filtered (1-30 Hz; $12 \mathrm{db}$ roll-off), baseline-corrected ( -200 to $0 \mathrm{~ms}$ before stimulus), and re-referenced to the average reference. The FRP was quantified 200-400 ms following reward feedback, and its peak and latency were scored for sites $\mathrm{Cz}, \mathrm{FCz}$, $\mathrm{Fz}$, and AFz, where the FRP is maximal (Potts et al., 2006; Foti et al., 2011; present study). To evaluate reward learning over the course of the experiment, primary analyses focused on FRP variables in early (block 1) and late (blocks 2 and 3 combined) phases of the task. Consistent with previous studies (Santesso et al., 2008, 2009), blocks 2 and 3 were combined based on behavioral data showing elevated response bias in blocks 2 and 3 relative to block 1 . Of note, we previously have referred to this component as the feedback-related negativity (FRN) (Santesso et al., 2008, 2009); recent findings, particularly those relying on principal component analyses to disentangle overlapping ERP components, suggest, however, that the FRN might in fact reflect a positive-going ERP deflection, which is reduced for unfavorable outcomes (Potts et al., 2006; Eppinger et al., 2008; Holroyd et al., 2008; Foti et al., 2011).

\section{Low-resolution electromagnetic tomography}

Low-resolution electromagnetic tomography (LORETA) (PascualMarqui et al., 1994) was used to estimate intracerebral current density underlying the FRP in blocks 2 and 3 (block 1 was excluded to allow participants to be exposed to the differential reinforcement schedule). Current density was computed as the linear weighted sum of the scalp electric potentials at each voxel $\left(N=2394\right.$; voxel resolution, $\left.7 \mathrm{~mm}^{3}\right)$ within 280-296 ms after feedback, which captured the FRP peak. The solution space was limited to cortical gray matter and hippocampi, as defined by the digitized Montreal Neurological Institute (MNI) template. For each subject, LORETA values were normalized to a total power of 1 and then log-transformed ( $\log 10)$ before analyses. To minimize type I error, statistical maps were thresholded at $p<0.01$ with minimum cluster size of 5 voxels.

\section{DNA}

DNA obtained from saliva samples was purified, extracted, and hydrated; it was stored at $-80^{\circ} \mathrm{C}$ when not in use (OG-100; OG-25; Oragene; DNA Genotek). CRHR1 SNPs occurring in $>5 \%$ of Caucasian populations $(n=57)$ were identified from the International HapMapProject Phase II B36 database (http://www.hapmap.org; International HapMap Consortium, 2005). The Tagger program (http://www.broad.mit.edu/mpg/tagger/; de Bakker et al., 2005) was used to determine the minimum set of SNPs necessary to capture or "tag" all HapMap SNPs (through linkage disequilibrium) with minor allele frequencies $>5 \%$ among Caucasians. Specifically, this tagging approach allowed us to select a limited number of SNPs that account for the entire genetic variation across a sequence of DNA by taking into account SNPs that are highly correlated with each other. SNPs tagged had a minimum $r^{2}$ of 0.8 with the tagging SNP (mean $r^{2}$ was 0.98$)$. The Tagger program identified 14 SNPs, which provided $100 \%$ coverage of CRHR $1 \pm 10 \mathrm{kbp}$ polymorphisms with a minor allele frequency $>5 \%$ in Caucasian individuals. Primers were designed using SpectroDESIGNER software (Sequenom). Following a PCR, an iPLEX massEXTEND reaction was performed. After baseline correction and peak identification, Sequenom SPECTROTYPER software was used to analyze resulting spectra. Concordance for duplicate DNA in the current 
sample $(n=17)$ was $100 \%$. No SNPs deviated from Hardy-Weinberg equilibrium (values of $p>0.55$ ).

In addition to rs12938031, three other CRHR1 SNPs and a haplotype have been associated with psychopathology and were also tested in secondary analyses (results available upon request). The Single Nucleotide Polymorphism Spectral Decomposition (SNPSpD) program (http:// gump.qimr.edu.au/general/daleN/SNPSpD/; Nyholt, 2004) was used to adjust the significance level due to multiple SNP comparisons. This spectral decomposition implements a Bonferroni correction that takes into account linkage disequilibrium between SNPs. Based on this procedure, $\alpha=0.017$ provided an appropriate significance threshold within the four CRHR1 SNPs tested with a priori hypotheses. This criterion was used to evaluate statistical significance of omnibus ANOVA effects involving genotype. In cases of significant ANOVA effects exceeding the threshold for multiple comparisons, post hoc tests were evaluated at $\alpha<0.05$.

\section{Statistical analyses}

Two sets of analyses were performed. In the first, the overall effects of stress on self-report, behavioral, scalp ERP, and source localization data were tested across the entire sample. In the second, the moderating effects of genotype were evaluated on a subsample with genetic data.

\section{Overall effects}

Stress manipulation. Condition (stress, no-stress) by Time (pre, during) ANOVAs were conducted for STAI and PANAS scales. A Condition by Block $(1,2,3)$ ANOVA was used to assess nonspecific skin conductance responses.

Behavioral data. Response bias and discriminability were analyzed with separate Condition by Block $(1,2,3)$ ANOVAs.

Scalp ERP data. Condition by Block [early phase (i.e., Block 1); late phase (i.e., Blocks 2 and 3)] by Site (AFz, Fz, FCz, Cz) ANOVAs were conducted on the amplitude and latency of the FRP in response to reward feedback.

Source localization. Using information from all channels, LORETA was used to estimate intracerebral current density underlying the FRP (Pascual-Marqui et al., 1994). Voxelwise paired $t$ tests compared intracerebral current density to reward feedback between the stress and nostress condition at the time of maximal FRP (280-296 ms after feedback).

\section{Stress by rs12938031 genotype}

For all analyses, the between-subject factor of Genotype was added. Due to findings from the overall analyses that FRP amplitudes were most reduced at the most anterior sites, genetic effects on the FRP were evaluated with Genotype by Condition by Block by Site (AFz, Fz) ANOVAs. For the sake of brevity, only effects involving Condition and Genotype are reported.

\section{Results}

\section{Overall effects}

Stress manipulation

Analyses of self-reported and skin conductance response data confirmed that the stress manipulation was successful. For both the STAI and the negative affect (NA) scale of the PANAS, main effects of Condition (both values of $F_{(1,73)}>23.74$; both values of $p<0.001)$ as well as significant Condition by Time interactions emerged $\left(\mathrm{STAI}, F_{(1,73)}=8.96, p<0.005 ; \mathrm{NA}, F_{(1,73)}=17.17, p<\right.$ 0.001 ; Fig. $1 A$ ). As intended, participants reported significantly elevated anxiety and negative affect during the stress condition (STAI, $43.67 \pm 9.93$; NA, $14.71 \pm 4.47$ ) compared with the nostress condition (STAI, $38.97 \pm 7.80$; NA, $12.50 \pm 2.74$ ) (both values of $t_{(74)}>4.73$; both values of $\left.p<0.001\right)$. Moreover, participants reported higher STAI and NA scores during the stress condition than just before it (STAI, $35.47 \pm 7.39$; NA, $11.80 \pm$ 1.79) (both values of $t_{(74)}>6.12$; both values of $p<0.001$ ). In addition, participants had a larger number of nonspecific skin conductance responses per minute in the stress $(2.89 \pm 2.35)$
A Overall Sample
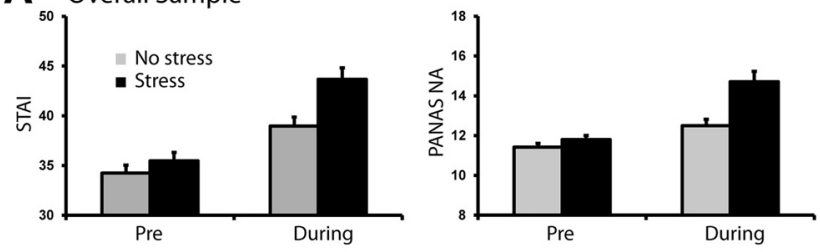

B A Homozygotes
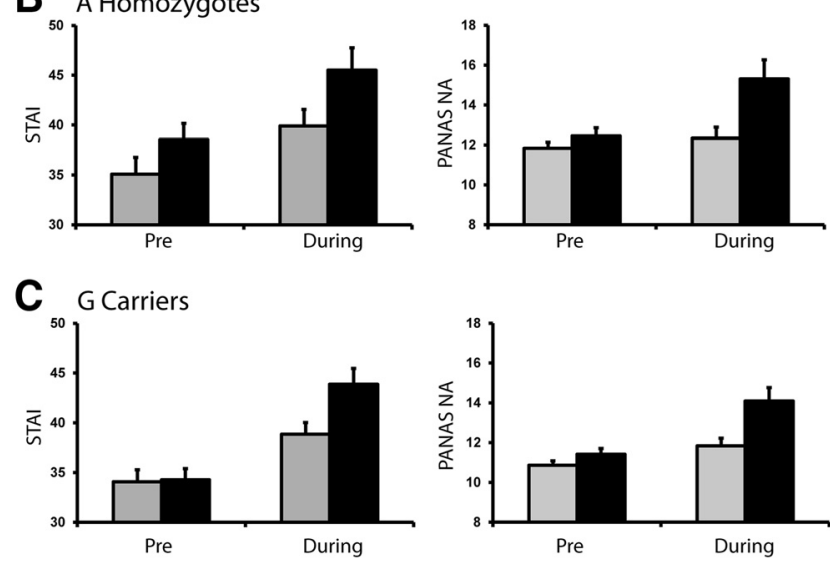

Figure 1. Effects of the acute stress manipulation on self-report measures of anxiety and negative affect. $A$, STAl scores, state version; NA score on the PANAS in the entire sample $(n=$ 74). $\boldsymbol{B}$, STAl and PANAS NA scores for rs 12938031 A homozygotes. C, STAl and PANAS NA scores for rs $12938031 \mathrm{G}$ carriers. No differences emerged across genotype groups. Pre, Assessment given before task administration. During, Assessment given directly following task administration with instructions to rate affect/anxiety while performing the task. Error bars indicate SEM.

relative to no-stress $(2.58 \pm 2.30)$ condition, but this difference was not significant $\left(t_{(62)}=1.37 ; p=0.18\right)$.

\section{Response bias}

A main effect of Block $\left(F_{(2,148)}=16.95 ; p<0.001\right)$ emerged due to increases in response bias over time [block $3(0.13 \pm 0.14)>$ block $2(0.08 \pm 0.12)>$ block $1(0.03 \pm 0.13)$; all values of $t_{(74)}>$ 2.71 , all values of $p<0.01]$; these findings confirm that the task successfully induced intended behavioral responses and that participants modulated behavior according to reward history. Critically, the main effect of Condition was significant due to lower response bias during the stress $(0.05 \pm 0.16)$ compared with the no-stress $(0.11 \pm 0.16)$ condition $\left(F_{(1,74)}=5.88\right.$; $p<0.02$; Fig. $2 A)$. Highlighting the specificity of these findings, the main effect of Condition and the Condition by Block interaction were not significant when considering discriminability (all values of $F_{(1,74)}<$ 0.96 ; all values of $p>0.33$ ). Thus, the acute stressor was not associated with a general drop in performance, but rather a reduced ability to modulate behavior according to reward history.

\section{Feedback-related positivity}

As hypothesized, the FRP was smaller and delayed during the stress $(0.02 \pm 1.61 \mu \mathrm{V} ; 302.84 \pm 34.10 \mathrm{~ms})$ relative to no-stress $(0.47 \pm$ $1.64 \mu \mathrm{V} ; 282.94 \pm 31.86 \mathrm{~ms})$ condition, as evident from significant main effects of Condition for both amplitude $\left(F_{(1,55)}=4.08 ; p<\right.$ $0.05)$ and latency $\left(F_{(1,55)}=15.14 ; p<0.001 ;\right.$ Fig. $\left.2 B\right)$. Findings were confirmed when evaluating only reward feedback following the more frequently reinforced stimulus (values of $F>4.50$; $p<0.05$ ).

\section{Source localization data}

As hypothesized, dorsal ACC activation (Brodmann areas 24/32) to reward feedback during the time period of the FRP was significantly lower in the stress relative to no-stress condition (Fig. $2 C$; 
$\left.t_{(55)}=3.42 ; p<0.001\right)$. This cluster extended into premotor cortex regions (Brodmann areas 6/8). An additional cluster encompassing orbitofrontal (OFC) and medial prefrontal cortex (PFC) (Brodmann areas 10/11) regions also showed reduced activation to reward feedback in the stress relative to no-stress condition $\left(t_{(55)}=3.45\right.$; $p<0.001$; Fig. $2 D)$. A complete list of regions can be found in Table 1 .

\section{Stress by rs12938031 genotype \\ Stress manipulation}

A main effect of Condition and a Condition by Time interaction emerged for both STAI and PANAS NA mirroring findings in the overall sample (all values of $F>$ 5.19; all values of $p<0.03$ ). There were no effects involving genotype for any stress manipulation variables (all values of $F<$ 1.76; all values of $p>0.19$ ), indicating that behavioral and ERP findings were not confounded by genotype differences in response to the stress manipulation (Fig. 1).

\section{Response bias}

In addition to the main effect of Condition $\left(F_{(1,52)}=6.34, p<0.02\right)$, which confirmed the effects of stress in the genetic subsample, the hypothesized Condition by Genotype interaction emerged $\left(F_{(1,52)}=10.95 ; p<\right.$ 0.003). Participants with A homozygosity at rs12938031-who display a depressive-like response to $\mathrm{CRH}$ infusion, blunted ventral striatal reactivity to positive feedback, and elevated depression following stress (Thode et al., 2011) — were characterized by stressinduced reward deficits (Fig. 3A). Post hoc paired $t$ tests revealed that A homozygotes had reduced response bias during the stress $(-0.01 \pm 0.15)$ relative to no-stress $(0.19 \pm 0.16)$ condition $\left(t_{(17)}=3.02 ; p<0.01\right)$. Moreover, relative to $\mathrm{G}$ carriers (no-stress, $0.07 \pm 0.16$; stress, $0.10 \pm$ $0.15)$, A homozygotes had elevated response bias during the nostress condition, but reduced response bias during the stress condition (both values of $t_{(52)}>2.41$; both values of $p<0.02$ ).

\section{Feedback-related positivity}

As for the entire sample, significant main effects of Condition emerged in the genetic subsample for both FRP voltage $\left(F_{(1,39)}=\right.$ $6.15 ; p<0.02)$ and latency $\left(F_{(1,39)}=6.91 ; p<0.02\right)$. Most importantly, a Condition by Genotype ANOVA revealed a significant interaction for both FRP amplitude $\left(F_{(1,39)}=8.93 ; p<0.005\right)$ and latency $\left(F_{(1,39)}=6.25 ; p<0.017 ;\right.$ Fig. $\left.3 B, C\right)$, indicating that groups significantly differed in their relative responses under the two conditions. As hypothesized, A homozygotes had a smaller (i.e., more negative) and delayed FRP during the stress relative to the no-stress condition (both values of $t_{(11)}>2.22$; values of $p<0.05$ ); for $\mathrm{G}$ carriers, FRP amplitude and latency did not differ between conditions (both values of $t_{(28)}<0.64$; values of $p>0.53$ ). Unpaired $t$ tests revealed no group differences under stress (both values of $t_{(39)}<$ 0.64 ; values of $p>0.53)$. In the no-stress condition, A homozygotes had a larger and earlier FRP relative to $G$ carriers (both values of $t_{(39)}>2.14$; values of $\left.p<0.04\right)$.
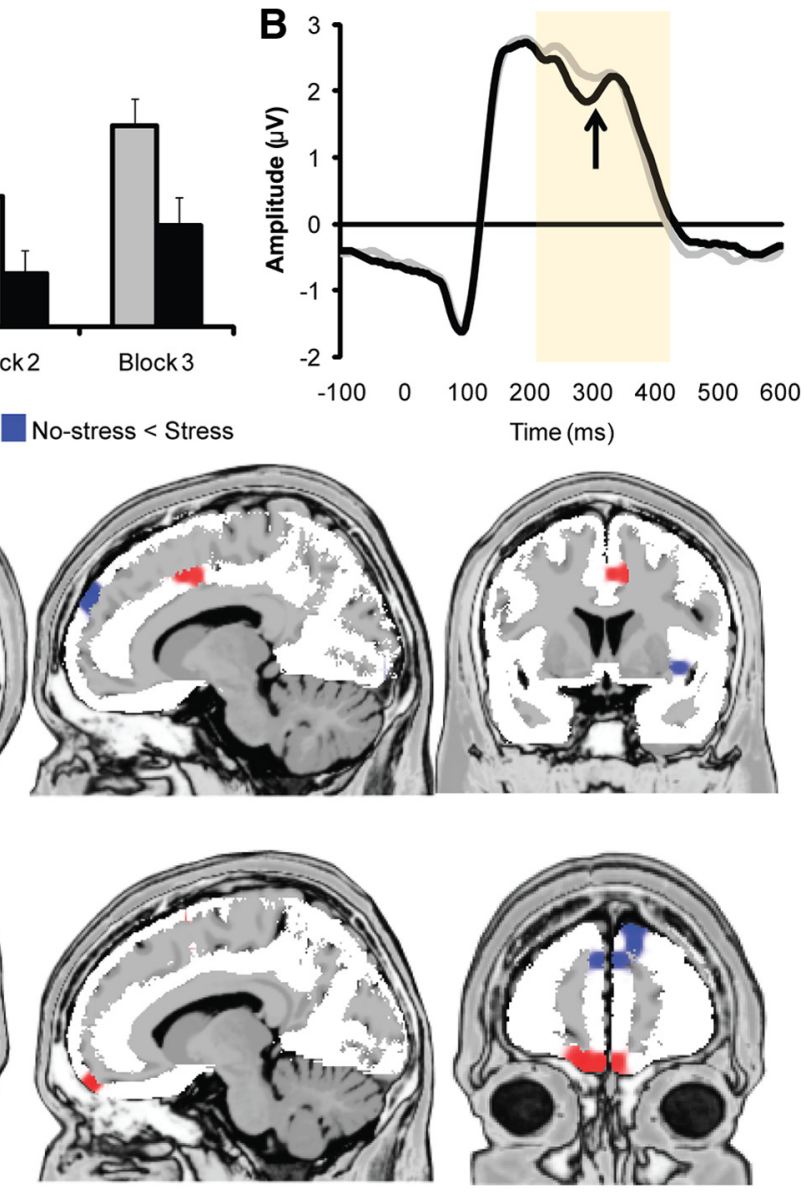

Figure 2. Overall effects (i.e., regardless of genotype) of the acute stress manipulation on response bias $(n=75)(\boldsymbol{A})$, FRP in response to reward feedback (see arrow; $n=56)(\boldsymbol{B})$, and LORETA data (calculated $280-296$ ms after reward feedback) $(n=56)$ dorsal ACC and OFC, respectively). LORETA statistical maps displayed on the MNI template and thresholded at $p<0.01$ (minimum cluster size, 5 voxels). Error bars indicate SEM.

Table 1. List of voxels exceeding statistical threshold from the LORETA analyses testing the contrast (no-stress-stress) across the entire sample $(n=56)$

\begin{tabular}{lrrrr}
\hline Location & \multicolumn{1}{c}{$y$} & \multicolumn{1}{c}{$z$} & $t$ value \\
\hline Orbital gyrus & -10 & 59 & -20 & 2.90 \\
Cingulate gyrus & 11 & 10 & 43 & 2.80 \\
Cingulate gyrus & -3 & 10 & 43 & 2.77 \\
Superior frontal gyrus & 4 & 10 & 64 & 2.77 \\
Superior frontal gyrus & 4 & 10 & 57 & 2.77 \\
Insula & 39 & 3 & -6 & -2.71 \\
Medial frontal gyrus & -3 & 59 & 22 & -2.88 \\
Superior temporal gyrus & 53 & 10 & -13 & -2.99 \\
Middle occipital gyrus & 53 & -74 & 1 & -3.04 \\
Superior temporal gyrus & 60 & -32 & 8 & -3.15 \\
Inferior temporal gyrus & 67 & -25 & -20 & -3.18 \\
Superior frontal gyrus & 11 & 59 & 36 & -3.18 \\
Fusiform gyrus & 53 & -60 & -20 & -3.42 \\
Middle temporal gyrus & 53 & -53 & -13 & -3.42 \\
Inferior parietal lobule & -52 & -67 & 43 & -3.44 \\
\hline
\end{tabular}

Note: The $x y z$ coordinates are in MNI space. Positive $t$ values reflect elevated activation during the no-stress relative to stress condition.

Statistical threshold: $p<0.01$; minimum cluster size, 5 voxels.

\section{Source localization}

Voxelwise Condition by Genotype ANOVAs revealed significant interactions for OFC and medial PFC regions (values of $p<$ $0.01)$. Follow-up voxelwise paired $t$ tests indicated that A ho- 



Figure 3. Significant Genotype by Condition interactions for rs 12938031 . Relative to $G$ carriers, A homozygotes showed significantly larger stress-induced behavioral reward deficits ( $G$ carriers, $n=36 ; \mathrm{AA}, n=18)(\boldsymbol{A})$, and FRP abnormalities (smaller and delayed FRP; $G$ carriers, $n=29 ; A A, n=12)(\boldsymbol{B}, \boldsymbol{C}$. $\boldsymbol{D}$, Relative to the no-stress condition, A homozygotes $(n=12)$ showed significantly reduced medial PFC/OFC activation in the stress condition 280-296 ms after reward feedback (see regions highlighted in red). $\boldsymbol{E}$, Relative to $G$ carriers $(n=29)$, A homozygotes showed significantly larger rostral ACC and ventromedial PFC activation under the no-stress condition $280-296 \mathrm{~ms}$ after reward feedback (see regions highlighted in red). No differences in ACC activity emerged under the stress condition. LORETA statistical maps displayed on the MNI template and thresholded at $p<0.01$ (minimum cluster size, 5 voxels). Error bars indicate SEM.

Table 2. Self-report measures by genotype at rs 12938031

\begin{tabular}{lcccr}
\hline Variable & A homozygote & G carrier & $t$ value & $p$ value \\
\hline PSS & $18.00 \pm 5.75$ & $17.27 \pm 5.78$ & $0.43^{a}$ & 0.67 \\
MASQ GDA & $16.29 \pm 4.65$ & $14.75 \pm 3.01$ & $1.46^{b}$ & 0.15 \\
MASQ AA & $18.71 \pm 2.49$ & $18.64 \pm 2.09$ & $0.10^{b}$ & 0.92 \\
MASQ GDD & $17.65 \pm 3.41$ & $16.31 \pm 4.55$ & $1.08^{b}$ & 0.29 \\
MASQ AD & $59.85 \pm 10.26$ & $54.21 \pm 7.79$ & $2.22^{b}$ & $<0.04$ \\
BDI-II & $2.00 \pm 2.43$ & $2.89 \pm 4.52$ & $0.77^{b}$ & 0.44
\end{tabular}

Values represent mean \pm SD. PSS, Perceived Stress Scale; MASQ, Mood and Anxiety Symptom Questionnaire; GDA, general distress anxiety; $A A$, anxious arousal; $G D D$, general distress depression; $A D$, anhedonic depression; $B D I-I$, Beck Depression Inventory II.

${ }^{a} \mathrm{df}=52$.

${ }^{b} \mathrm{df}=51$.

mozygotes had reduced OFC/medial PFC (Brodmann areas 10/ 11) activation to reward feedback during the stress relative to no-stress condition $(p<0.01)$ (Fig. 3D), while G carriers showed no differences between conditions. Unpaired $t$ tests revealed no group differences in the stress condition $(p>0.07)$. In the nostress condition, A homozygotes had larger activation than $\mathrm{G}$ carriers in the medial PFC, OFC, ACC (Brodmann areas 10/11/ $32 ; p<0.01$ ) (Fig. 3E).

\section{Control analyses}

A homozygotes reported elevated MASQ anhedonic depression scores (Table 2). Importantly, ANCOVA analyses entering anhedonic depression scores as covariates confirmed the Genotype by Condition interaction for response bias $\left(F_{(1,50)}=8.00\right.$; $p<$
$0.01)$, FRP amplitude $\left(F_{(1,37)}=7.58 ; p<\right.$ $0.01)$, and FRP latency $\left(F_{(1,37)}=5.01 ; p=\right.$ $0.031)$, although the latency finding did not survive correction for multiple SNP comparisons.

\section{Discussion}

This study examined how a functional CRHR1 genetic polymorphism (rs12938031) and acute stress influence reward learning in healthy Caucasian women. Replicating independent findings (Bogdan and Pizzagalli, 2006), acute stress reduced participants' ability to develop a response bias toward a more frequently rewarded stimulus. Moreover, under stress, the FRP was blunted and delayed and dorsal ACC activation to reward feedback was reduced. Finally, an interaction qualified these main effects, wherein A homozygotes were more susceptible to stress-induced reward learning deficits.

\section{CRHR1 genotype, stress, and reward learning}

As in prior studies (Bogdan and Pizzagalli, 2006; Liu et al., 2011), reduced response bias under stress emerged in the context of no difference in discriminability, suggesting that acute stress did not affect global performance. Instead, under stress, participants were less able to modulate behavior as a function of reinforcement history. These data support a wealth of animal (Anisman and Matheson, 2005; Willner, 2005) and growing human (Berenbaum and Connelly, 1993; Bogdan and Pizzagalli, 2006; Pizzagalli et al., 2007) research suggesting that stress may induce reward processing dysfunction.

Alongside stress-induced behavioral deficits, we report for the first time that participants had a smaller and delayed FRP as well as reduced ACC activation to reward feedback under stress. Notably, prior studies indicate that FRP amplitudes reflect positive learning signals (positive prediction errors), which have been localized to dorsal ACC (Potts et al., 2006) and preliminarily to striatal (Foti et al., 2011) regions implicated in reinforcement learning. Thus, both the behavioral and FRP findings may reflect a reduced ability to integrate reinforcement history under stress. Along similar lines, prior studies have reported that the FRP is reduced to unexpected outcomes (Hajcak et al., 2007; Oliveira et al., 2007) or when stimulus-outcome representations rely upon external feedback, particularly during initial learning phases (Müller et al., 2005; Heldmann et al., 2008). Accordingly, smaller (i.e., more negative) FRP amplitudes under stress might reflect heightened reliance on external feedback and/or a reduced ability to predict positive feedback resulting in greater expectancy violation. Fitting this interpretation and prior findings (Müller et al., 2005), as learning progressed, participants showed significantly larger FRP amplitude and shorter latency (results available upon request). The current results nicely dovetail with prior findings from our laboratory showing smaller FRP to reward feedback in participants (1) with low reinforcement learning (Santesso et al., 2008), and (2) receiving a pharmacological challenge hypothesized to reduce phasic DA signaling (Santesso et al., 2009). 
A wealth of literature emphasizes frontostriatal networks in reinforcement-guided decision making (Haber and Knutson, 2010). Dorsal ACC regions, in particular, play a key role in representing reinforcement history and integrating action-outcome associations in the service of guiding adaptive goal-directed behavior (Kennerley et al., 2006; Santesso et al., 2008). The OFC, conversely, has been implicated in the representation of affective value for reinforcers (Rolls and Grabenhorst, 2008). Interestingly, consistent with prior reports implicating the ACC in FRP generation (Potts et al., 2006), we observed stress-induced reductions in ACC and OFC activation during the FRP, which might index diminished expected value and lead to suboptimal reinforcement-related behavioral adjustments.

Importantly, behavioral, ERP and source localization main effects of stress were qualified by CRHR1 genotype by stress interactions. A homozygotes at rs12938031-who showed depressive-like blunted ACTH and cortisol responses to $\mathrm{CRH}$ infusion, reduced reward-related activation to positive feedback, heightened threat-related amygdala activation, and elevated depression following stress in a recent study (Thode et al., 2011) had blunted reward learning, a smaller and delayed FRP, as well as reduced medial $\mathrm{PFC/OFC}$ activation to reward delivery under stress-but not no-stress - conditions (even after correcting for multiple testing). In light of research suggesting that (1) CRHR1 influences DA function (Beckstead et al., 2009), and (2) reduced activity within the medial PFC in response to uncontrollable stress is associated with depressive- and anxiety-like behavior (Maier et al., 2006), A homozygotes may be more susceptible to stress-induced DA dysfunction discussed below, perhaps due to a reduced perception of control over stressors. The current findings indicate that CRHR1 genotype (i.e., rs12938031) may promote the development of anhedonia in the face of stress, providing initial evidence for a promising candidate mechanism explaining associations between this genotype, stress, and depression (Thode et al., 2011). Interestingly, during the no-stress condition, A homozygosity was associated with elevated reward learning, FRP, and enhanced medial prefrontal response to reward feedback. Taken together, these findings of stress-induced deficits but basal enhancements are consistent with recent theoretical work suggesting that genetic variants associated with "risk" following aversive experience may instead provide plasticity to environmental factors, for better or worse (Belsky et al., 2009).

\section{Candidate neurological mechanisms}

Although we have now observed that stress is associated with reduced reinforcement learning in psychiatrically healthy participants in four independent studies (Bogdan and Pizzagalli, 2006; Pizzagalli et al., 2007; Nikolova et al., 2011; present study), the precise mechanisms underlying this phenomenon are unknown. This study provides novel etiological insights by showing that stress reduces the FRP and that behavioral and neural effects are dependent upon CRHR1 genotype.

An influential theory proposes that the ACC is tonically inhibited by afferent DA inputs originating from the basal ganglia and that more negative FRP amplitudes arise from phasic DA dips disinhibiting ACC cells (Holroyd and Coles, 2002). Critically, in nonhuman animals, acute stress enhances tonic DA levels (Cabib and Puglisi-Allegra, 1996) and tonically elevated DA reduces phasic DA bursts (Bilder et al., 2004). Thus, although speculative, acute stress may result in tonically elevated DA levels (and thus, inhibited ACC activity) but reduced DA bursts resulting in reduced positive prediction errors and deficient reinforcement learning (and thus, more negative and delayed FRP to rewards). We note that an identical pattern of findings (i.e., reduced response bias, smaller FRP, and reduced dorsal ACC activation to reward feedback) emerged in a study in which a single, low-dose of the DA agonist pramipexole-thought to reduce phasic DA burst to reward feedback through autoreceptor activation-was administered to healthy participants (Santesso et al., 2009). Together, these findings indicate that a variety of manipulations resulting in reduced phasic DA bursts may reduce reward learning in humans. Future studies using neuroimaging techniques allowing for the assessment of DA release (e.g., raclopride-based PET displacement) are needed to test this hypothesis.

A homozygosity at rs12938031 may potentiate these effects. Animal research shows that $\mathrm{CRH}$ infusion, primarily through activation of CRHR1 receptors, elevates DA levels, similar to acute stress (Wanat et al., 2008). Thus, the enhanced CRHR1 expression characteristic of rs12938031 A homozygotes combined with acute stress-induced elevations of tonic dopamine may compound one another to leave A homozygotes particularly sensitive to stress-induced reward learning deficits. Based on animal data, we speculate that acute stress and CRHR1 genotype may negatively impact the formation of new stimulus-reward associations but enhance responsiveness to habitual reward. Indeed, research suggests that $\mathrm{CRH}$ administration and stressors enhance response to reward cues that are already formed; for example, individuals who abuse drugs or who have already been conditioned to stimuli show greater responses to these stimuli under stress (Brady et al., 2009; Schwabe et al., 2011) consistent with the notion that stress may increase habitual responding over goal-directed action (Schwabe and Wolf, 2011a,b). Future research is needed to evaluate the effects of stress on the formation of both new and established reward-stimulus associations.

\section{Limitations and conclusions}

The limitations of this study deserve attention. First, while the sample size is relatively large for an ERP investigation, it is small for a genetic study, and its generalizability is limited by the inclusion of Caucasian female participants only. This issue was compounded by the loss of subjects for genetic and FRP analyses. As a result, these data await replications from larger and more heterogeneous samples. A second limitation is the laboratory-based nature of the stress manipulation. While the use of a laboratory stressor is an experimental strength, its ecological validity is limited and it cannot be directly compared with real-life stressors. Notably, we recently found that perceived stress about an upcoming naturalistic stressor was associated with reduced response bias (Nikolova et al., 2011), suggesting that real-life and laboratory stressors similarly reduce reward learning. Third, endocrine measures of stress responsiveness were not assessed; it would be particularly interesting for future research to examine whether such measures further mediate the effects reported here. Finally, while the stress condition elicited intended affective and peripheral psychophysiological responses, the difference between conditions in skin conductance responses was not significant. Overall, this pattern highlights a mild stress elicitation, raising the possibility that effects might be larger with more severe and chronic stressors. Despite these limitations, the current findings replicate prior data that stress disrupts reward learning and show for the first time that individuals with certain CRHR1 polymorphisms (e.g., rs12938031) are particularly prone to stressinduced reward abnormalities. Future studies will be required to evaluate whether the emergence of stress-induced anhedonia, particularly in individuals at putatively increased genetic risk, 
might explain links between stress and various forms of psychopathology, including depression.

\section{Notes}

Supplemental material for this article is available online at http:// cdasr.mclean.harvard.edu/CRHR1. This material provides information regarding linkage disequilibrium for all CRHR1 SNPs typed, results for three secondary SNPs, and ancillary results. Particularly, control ANOVAs revealed no stress effects on ERP components time-locked to the rich/lean stimuli, highlighting the specificity of the main FRP findings. Moreover, analyses confirmed that the task elicited the intended effects, including higher accuracy and shorter reaction time for the rich, relative to lean, stimulus. Lastly, two SNPs with a priori hypotheses (i.e., rs4076452 and rs10445364) showed Genotype by Condition interactions for response bias that survive correction for multiple testing. This material has not been peer reviewed.

\section{References}

Anisman H, Matheson K (2005) Stress, depression, and anhedonia: caveats concerning animal models. Neurosci Biobehav Rev 29:525-546.

Beck AT, Steer RA, Brown GK (1996) Beck Depression Inventory Manual. San Antonio, TX: The Psychological Corporation.

Beckstead MJ, Gantz SC, Ford CP, Stenzel-Poore MP, Phillips PE, Mark GP, Williams JT (2009) CRF enhancement of GIRK channel-mediated transmission in dopamine neurons. Neuropsychopharmacology 34: 1926-1935.

Belsky J, Jonassaint C, Pluess M, Stanton M, Brummett B, Williams R (2009) Vulnerability genes or plasticity genes? Mol Psychiatry 14:746-754.

Berenbaum H, Connelly J (1993) The effect of stress on hedonic capacity. J Abnorm Psychol 102:474-481.

Bilder RM, Volavka J, Lachman HM, Grace AA (2004) The catechol-Omethyltransferase polymorphism: relations to the tonic-phasic dopamine hypothesis and neuropsychiatric phenotypes. Neuropsychopharmacology 29:1943-1961.

Binder EB, Nemeroff CB (2010) The CRF system, stress, depression and anxiety-insights from human genetic studies. Mol Psychiatry 15:574-588.

Bogdan R, Pizzagalli DA (2006) Acute stress reduces reward responsiveness: implications for depression. Biol Psychiatry 60:1147-1154.

Bogdan R, Pizzagalli DA (2009) The heritability of hedonic capacity and perceived stress: a twin study evaluation of candidate depressive phenotypes. Psychol Med 39:211-218.

Bogdan R, Perlis RH, Fagerness J, Pizzagalli DA (2010) The impact of mineralocorticoid receptor iso/val genotype (rs5522) and stress on reward learning. Genes Brain Behav 9:658-667.

Brady KT, McRae AL, Moran-Santa Maria MM, DeSantis SM, Simpson AN, Waldrop AE, Back SE, Kreek MJ (2009) Response to corticotropinreleasing hormone infusion in cocaine-dependent individuals. Arch Gen Psychiatry 66:422-430.

Cabib S, Puglisi-Allegra S (1996) Stress, depression and the mesolimbic dopamine system. Psychopharmacology 128:331-342.

Cohen S, Kamarck T, Mermelstein R (1983) A global measure of perceived stress. J Health Soc Behav 24:385-396.

de Bakker PI, Yelensky R, Pe'er I, Gabriel SB, Daly MJ, Altshuler D (2005) Efficiency and power in genetic association studies. Nat Genet 37:1217-1223.

Dickerson SS, Kemeny ME (2004) Acute stressors and cortisol responses: a theoretical integration and synthesis of laboratory research. Psychol Bull 130:355-391.

Dillon DG, Holmes AJ, Birk JL, Brooks N, Lyons-Ruth K, Pizzagalli DA (2009) Childhood adversity is associated with left basal ganglia dysfunction during reward anticipation. Biol Psychiatry 66:206-213.

Dreher JC, Kohn P, Kolachana B, Weinberger DR, Berman KF (2009) Variation in dopamine genes influences responsivity of the human reward system. Proc Natl Acad Sci U S A 106:617-622.

Eppinger B, Kray J, Mock B, Mecklinger A (2008) Better or worse than expected? Aging, learning, and the ERN. Neuropsychologia 46:521-539.

First MB, Spitzer RL, Gibbon M, Williams JBW (2002) Structured clinical interview for DSM-IV axis I disorders. New York: Biometrics Research, New York State Psychiatric Institute.

Foti D, Weinberg A, Dien J, Hajcak G (2011) Event-related potential activity in the basal ganglia differentiates rewards from nonrewards: temporospatial principal components analysis and source localization of the feedback negativity. Hum Brain Mapp. Advance online publication. Retrieved August 3, 2011. doi:10.1002/hbm.21182.

Gottesman II, Gould TD (2003) The endophenotype concept in psychiatry: etymology and strategic intentions. Am J Psychiatry 160:636-645.

Grillon C, Ameli R, Merikangas K, Woods SW, Davis M (1993) Measuring the time course of anticipatory anxiety using the fear-potentiated startle reflex. Psychophysiology 30:340-346.

Haber SN, Knutson B (2010) The reward circuit: linking primate anatomy and human imaging. Neuropsychopharmacology 35:4-26.

Hajcak G, Moser JS, Holroyd CB, Simons RF (2007) It's worse than you thought: the feedback negativity and violations of reward prediction in gambling tasks. Psychophysiology 44:905-912.

Hasler G, Drevets WC, Manji HK, Charney DS (2004) Discovering endophenotypes for major depression. Neuropsychopharmacology 29:17651781.

Hauger RL, Risbrough V, Brauns O, Dautzenberg FM (2006) Corticotropin releasing factor $(\mathrm{CRF})$ receptor signaling in the central nervous system: new molecular targets. CNS Neurol Disord Drug Targets 5:453-479.

Hautus MJ (1995) Corrections for extreme proportions and their biasing effects on estimated values of $d^{\prime}$. Behav Res Methods Instrum Comput 27:46-51.

Heldmann M, Rüsseler J, Münte TF (2008) Internal and external information in error processing. BMC Neurosci 9:33.

Holroyd CB, Coles MG (2002) The neural basis of human error processing: reinforcement learning, dopamine, and the error-related negativity. Psychol Rev 109:679-709.

Holroyd CB, Pakzad-Vaezi KL, Krigolson OE (2008) The feedback correctrelated positivity: sensitivity of the event-related brain potential to unexpected positive feedback. Psychophysiology 45:688-697.

International HapMap Consortium (2005) A haplotype map of the human genome. Nature 437:1299-1320.

Jung TP, Makeig S, Westerfield M, Townsend J, Courchesne E, Sejnowski T] (2000) Removal of eye activity artifacts from visual event-related potentials in normal and clinical subjects. Clin Neurophysiol 111:1745-1758.

Kennerley SW, Walton ME, Behrens TE, Buckley MJ, Rushworth MF (2006) Optimal decision making and the anterior cingulate cortex. Nat Neurosci 9:940-947.

Liu WH, Chan RC, Wang LZ, Huang J, Cheung EF, Gong QY, Gollan JK (2011) Deficits sustaining reward responses in subsyndromal and syndromal major depression. Prog Neuropsychopharmacol Biol Psychiatry 35:1045-1052.

Maier SF, Amat J, Baratta MV, Paul E, Watkins LR (2006) Behavioral control, the medial prefrontal cortex, and resilience. Dialogues Clin Neurosci 8:397-406.

McCarthy D, Davison M (1979) Signal probability, reinforcement and signal detection. J Exp Anal Behav 32:373-386.

Müller SV, Möller J, Rodriguez-Fornells A, Münte TF (2005) Brain potentials related to self-generated and external information used for performance monitoring. Clin Neurophysiol 116:63-74.

Nikolova YS, Bogdan R, Pizzagalli DA (2011) Perception of a naturalistic stressor interacts with 5-HTTLPR/rs25531 genotype and gender to impact reward responsiveness. Neuropsychobiology, in press.

Nolen-Hoeksema S, Larson J, Grayson C (1999) Explaining the gender difference in depressive symptoms. J Pers Soc Psychol 77:1061-1072.

Nyholt DR (2004) A simple correction for multiple testing for SHPs in linkage disequilibrium with each other. Am J Hum Genet 74:765-769.

Oliveira FT, McDonald JJ, Goodman D (2007) Performance monitoring in the anterior cingulate is not all error related: expectancy deviation and the representation of action-outcome associations. J Cogn Neurosci 19:1994-2004.

Pascual-Marqui RD, Michel CM, Lehmann D (1994) Low resolution electromagnetic tomography: a new method for localizing electrical activity in the brain. Int J Psychophysiol 18:49-65.

Pizzagalli DA, Jahn AL, O'Shea JP (2005) Toward an objective characterization of ananhedonicphenotype: a signal-detection approach. Biol Psychiatry $57: 319-327$.

Pizzagalli DA, Bogdan R, Ratner KG, Jahn AL (2007) Increased perceived stress is associated with blunted hedonic capacity: potential implications for depression research. Behav Res Ther 45:2742-2753.

Pizzagalli DA, Iosifescu D, Hallett LA, Ratner KG, Fava M (2008) Reduced 
hedonic capacity in major depressive disorder: evidence from a probabilistic reward task. J Psychiatr Res 43:76-87.

Potts GF, Martin LE, Burton P, Montague PR (2006) When things are better or worse than expected: the medial frontal cortex and the allocation of processing resources. J Cogn Neurosci 18:1112-1119.

Rolls ET, Grabenhorst F (2008) The orbitofrontal cortex and beyond: from affect to decision-making. Prog Neurobiol 86:216-244.

Santesso DL, Dillon DG, Birk JL, Holmes AJ, Goetz E, Bogdan R, Pizzagalli DA (2008) Individual differences in reinforcement learning: behavioral, electrophysiological, and neuroimaging correlates. Neuroimage 42:807816.

Santesso DL, Evins AE, Frank MJ, Schetter EC, Bogdan R, Pizzagalli DA (2009) Single dose of a dopamine agonist impairs reinforcement learning in humans: evidence from event-related potentials and computational modeling of striatal-cortical function. Hum Brain Mapp 30:1963-1976.

Schwabe L, Wolf OT (2011a) Stress increases behavioral resistance to extinction. Psychoneuroendocrinology. Advance online publication. Retrieved August 3, 2011. doi:10.1016/j.psyneuen.2011.02.002.

Schwabe L, Wolf OT (2011b) Stress-induced modulation of instrumental behavior: from goal-directed to habitual control of action. Behav Brain Res 219:321-328.

Schwabe L, Dickinson A, WolfOT (2011) Stress, habits, and drug addiction: a psychoneuroendocrinological perspective. Exp Clin Psychopharmacol 19:53-63.
Spielberger CD, Gorsuch RL, Lushere RE (1970) Manual of the State-Trait Anxiety Inventory. Palo Alto, CA: Consulting Psychologists Press.

Takahashi LK (2001) Role of $\mathrm{CRF}_{1}$ and $\mathrm{CRF}_{2}$ receptors in fear and anxiety. Neurosci Biobehav Rev 25:627-636.

Thode K, Walss-Bass C, Hariri AR, Olvera R, Muñoz K, Qureshi N, Beuten J, Gelfond J, Maher B, Dahl R, Birmaher B, Ryan N, Williamson DE (2011) Functional evidence implicating the role of the corticotropin-releasing hormone receptor 1 in development of stress-related disorders. Am J Psychiatry, in press.

Tripp G, Alsop B (1999) Sensitivity to reward frequency in boys with attention deficit hyperactivity disorder. J Clin Child Psychol 28:366-375.

Wanat MJ, Hopf FW, Stuber GD, Phillips PE, Bonci A (2008) Corticotropin-releasing factor increases mouse ventral tegmental area dopamine neuron firing through a protein kinase $\mathrm{C}$-dependent enhancement of lh. J Physiol 586:2157-2170.

Watson D, Clark LA, Tellegen A (1988) Development and validation of brief measures of positive and negative affect: the PANAS scales. J Pers Soc Psychol 54:1063-1070.

Watson D, Clark LA, Weber K, Assenheimer JS, Strauss ME, McCormick RA (1995) Testing a tripartite model: II. Exploring the symptom structure of anxiety and depression in student, adult, and patient samples. J Abnorm Psychol 104:15-25.

Willner P (2005) Chronic mild stress (CMS) revisited: consistency and behavioral neurobiological concordance in the effects of CMS. Neuropsychobiology 52:90-110. 